particles. As a result of these collisions a range of small-sized particles would be formed, and those with dimensions of the order of $10^{-5} \mathrm{~cm}$. would be driven off from the comet by the sun's radiation pressure; in this way a comet would produce tails indefinitely at each return to perihelion.

One important effect may be mentioned. Differences of period in the particles must result in their distribution around the orbit of the comet, and in some cases these particles are responsible for meteor streams. In addition, collisions could lead to a resistance which would have effects similar to the Poynting-Robertson effect, though differing quantitatively, thus decreasing the perihelion distances of comets and accelerating their velocities.

This is described in a paper by $H$. P. Robertson, under the title, "Dynamical Effects of Radiation in the Solar System"s. Poynting showed that the absorption and re-radiation of solar radiation by small bodies produced a tangential drag, decreasing the angular momentum of the particles and causing them to spiral inwards towards the sun ; and Robert. son examined the problem from the point of view of relativity. He obtained expressions for the retarding force which differed from those of Poynting and Larmor, and he admitted that the values caused by the drag could not be reconciled at the same time between the various elements. Lyttleton suggests that the further mechanism provided by his theory, combined with the Poynting-Robertson effect, might partly solve the difficulty that Robertson met in dealing with Encke's comet. It is hoped that Lyttleton will pursue this important subject later.

${ }^{1}$ Mon. Not. Roz. Astro. Soc., 111. 3 (1951).

${ }^{2}$ Mon. Not. Roy. Astro. Soc., 108, 6 (1948); see also Nature, 164, 119 (1949).

${ }^{3}$ Mon. Not. Roy. Astro. Soc., 97. 436 (1937).

\section{ELECTRONIC TELEPHONE EXCHANGES}

A agLEPHONE exchange is basically a switch or
subscribers are connected together for the conduct
of a telephone conversation. A manual exchange
system is one in which human operators carry out
the interconnexions between the subscribers ; but
the idea was conceived some sixty years ago that
this work could be conducted automatically by means
of electromagnetic switches operated by impulses
sent by the subscriber initiating the telephone call.
This conception has been developed notably in the
past thirty years or so; and alaut half a dozen
systems of automatic telephone exchange have
achieved large-scale use in different parts of the
world. During this period of development the
thermionic valve amplifier, or repeater, has also been
applied and has become a normal piece of equipment
in any trunk or long-line telephone system. More
recently, attention has been given in Great Britain
and other countries to the possibilities of automatic
telephone exchanges constructed with electronic
switches, in order to determine whether exchanges
cheaper and more reliable than the present types
using electro-mechanical switches may thereby be
produced.

At a meeting of the Institution of Electrical Engineers on March 13, a very interesting paper entitled "Electronic Telephone Exchanges" was read by T. H. Flowers, of the Post Office Research Station, Dollis Hill. The author approached his subject in a broad manner, and did not merely limit his theme to a simple problem of replacing a number of electromechanical switches by the corresponding valve assemblies carrying out the same individual functions. The paper considered the problem of line interconnexion anew from first principles, and then discussed some of the practical forms which electronic exchanges might take, the possibilities and characteristics of such exchanges, and--very briefly-the questions of cost and reliability. The multiplex switching arrangements required can make use of carrier-frequency or pulse techniques to obtain frequency- or time-division systems respectively. At the reading of the paper a demonstration was given of the operation of a model three-line exchange using trains of pulses for interconnexion, line-signalling and speech transmission between the subscribers' installations. Speech frequencies of $300-4,000 \mathrm{c} / \mathrm{s}$. can be transmitted with a pulse-repetition frequency of $10 \mathrm{kc} . / \mathrm{s}$. for the trains of pulses. For a switch dealing with a hundred channels, the time-spacing between the channels is then $1 \mu$ sec., and a pulse width of $0.3 \mu$ sec. is desirable. As Mr. Flowers points out in his paper, transmission gain and loss, variation of loss, and distortion are features of electronic connector switches not found in existing types using metal-to-metal contacts; but, on the whole, better speech transmission could be given by electronic systems using the connector switches described in the paper.

In the discussion, which followed the reading of the paper, several speakers emphasized the need for caution before too hurriedly adopting these new techniques, since cost and effective life are major items for consideration. A modern telephone exchange is expected to last for at least twenty-five years, and, as Mr. Flowers pointed out, it would be necessary to guarantee at least a five-year life for the components, including the valves, if the effect of failures on the service and the cost of replacing faulty parts are not to be prohibitive. The meeting provided a very useful introductory outline on the possibilities of electronic automatic telephone exchanges, and, whatever may be the outcome, there appears to be enough encouraging evidence to justify proceeding with the development of such exchanges.

\section{COSMIC-RAY EXPEDITION TO THE HIMALAYAS}

BY K. B. MATHER

Physics Department, University of Ceylon, Colombo

THE University of Ceylon recently sponsored a cosmic-ray expedition to the Garhwal Himalayas. Early in 1951 various high-altitude experiments were designed by Dr. V. Appapillai and me, and a grant from the research committee of the University was obtained to enable these to be carried out. Accounts of the experimental work will be published in due course as the results become available. The purpose of the present note is to communicate some general information concerning the expedition, which, it is felt, will be of interest to cosmic-ray physicists and perhaps to others. 